\title{
Projeção da Estrutura Diamétrica de Grupos Ecológicos em uma Floresta Ombrófila Mista
}

\author{
Sebastião Machado ${ }^{1}$, Vinicius Cysneiros ${ }^{1}$, Rodrigo Geroni Nascimento ${ }^{1}$, \\ Ana Martins ${ }^{1}$, Luciane Schmidt ${ }^{1}$ \\ ${ }^{1}$ Departamento de Ciências Florestais, Universidade Federal do Paraná - UFPR, \\ Curitiba/PR, Brasil
}

\begin{abstract}
RESUMO
O presente estudo teve como objetivo avaliar os aspectos ecológicos e sucessionais de um fragmento de floresta subtropical via projeção da estrutura diamétrica de diferentes grupos ecológicos com uso da Matriz de Transição. Com base em medições de 2009 e 2012 de todas as árvores acima de $10 \mathrm{~cm}$ de DAP, foram realizadas projeções da distribuição diamétrica, bem como análise da dinâmica de três agrupamentos de espécies: Pioneiras; Não Pioneiras; e Floresta como um todo. Simulações da dinâmica dos agrupamentos foram feitas até 2027 após validação do método. O método de projeção se mostrou aderente em todos os casos, apresentando maior precisão quando utilizado o intervalo de classe de $5 \mathrm{~cm}$. As análises indicaram tendência de substituição gradual das espécies pioneiras, sugerindo avanço sucessional no fragmento florestal estudado, bem como eficiência do método para análise da dinâmica dos grupos ecológicos estudados.
\end{abstract}

Palavras-chave: dinâmica florestal, sucessão florestal, cadeia de Markov.

\section{Diametric Distribution Projection of Ecological Groups in a Mixed Tropical Forest}

\begin{abstract}
This study aimed to evaluate the ecological and successional aspects of a subtropical forest fragment by projection of the diameter distribution of different ecological groups, using transition matrix model. Based on measurements of 2009 and 2012 of all trees above $10 \mathrm{~cm}$ of DBH, projection of the diameter distribution as well as analysis of forest dynamic were made for three species groups: Pioneer; Non Pioneers, and the whole forest. Simulations of the dynamics of the groups were made until 2027 after method validation. The projection method was adherent in all cases, with higher precision when using the $5 \mathrm{~cm}$ class interval. The analysis made indicated a gradual substitution tendency of the pioneer species, suggesting a successional advance in the forest fragment under study, as well as an efficiency of the projection method for dynamics analysis of the ecological groups studied.
\end{abstract}

Keywords: forest dynamics, forest succession, chain of Markov. 


\section{INTRODUÇÃO}

As florestas naturais são dinâmicas, em aspectos temporais e espaciais, formando mosaicos de estágios sucessionais, cuja configuração depende de uma série de fatores ambientais e ecológicos associados às características ecofisiológicas das espécies. A dinâmica estrutural e florística das florestas são causadas por diversos fatores que propiciam heterogeneidade ambiental no espaço e no tempo, em uma ampla escala de ação (Nascimento et al., 2012). Nas florestas tropicais e subtropicais, a sucessão florestal é comumente caracterizada pela substituição gradual do predomínio de espécies pioneiras por espécies não pioneiras ou clímax (Richards, 1957), convergindo para um sistema mais complexo, com máxima biomassa e diversidade (Odum, 1969). Essas florestas podem progredir ou retroceder em diversidade estrutural e florística de acordo com distúrbios internos, como abertura de clareiras ou externos, como fogo e exploração seletiva (Whitmore, 1978; Pillar, 1994).

Para a compreensão desses processos de sucessão ecológica de florestas tropicais, é essencial o estudo das qualidades adaptativas e evolutivas das espécies quanto a suas estratégias de ocupação dos ambientes (Piña-Rodrigues et al., 1990; Paula et al., 2004). $\mathrm{O}$ agrupamento das espécies arbóreas em grupos ecológicos, bem como de classe de tamanho e formas de vida, são as técnicas mais utilizadas para entendimento da dinâmica de floresta, pois facilitam a compreensão dos processos de sucessão. As características ecológicas mais usadas são: ciclo de vida; reprodução; propagação; ritmo de crescimento; capacidade fotossintética, e; regeneração (Whitmore, 1989, 1990). Já o agrupamento via a distribuição diamétrica permite o entendimento dos processos de sucessão, propiciando informações sobre a regeneração natural, bem como da estrutura etária das populações (Harper, 1990; Paula et al., 2004; Canalez et al., 2006). Logo, o conhecimento da composição, estrutura e dinâmica da floresta em conjunto com as informações ecológicas das espécies fornece importantes evidências sobre a sucessão florestal, permitindo inferir sobre o estágio sucessional da floresta e adoção de boas ações de manejo e conservação dos seus recursos (Piña-Rodrigues et al., 1990; Pillar, 1994; Gandolfi et al., 1995; Maciel et al., 2003; Paula et al., 2004).
Os estudos sobre sucessão ecológica em ambientes florestais são baseados em inventário florestal contínuo, pois apenas com auxílio das informações obtidas nesses levantamentos é possível identificar os fatores que afetam a dinâmica da floresta, bem como utilizar essas informações para prognose de comportamento futuro por meio de modelos matemáticos.

O uso de modelos de predição e prognose facilitam a análise da dinâmica da estrutura diamétrica em florestas naturais, propiciando uma avaliação prévia dos processos de sucessão florestal futuros, fornecendo subsídios para a tomada de decisão antecipada quanto ao uso dos recursos florestais. A Cadeia de Markov ou Matriz de Transição consiste em um dos diversos processos estocásticos de predição para florestas tropicais. Esse tipo de modelo matemático é amplamente utilizado e difundido em estudos florestais de sistemas complexos relacionados à dinâmica, sucessão, crescimento e produção florestal (Alder, 1980; Buogiorno \& Michie, 1980; Shuggart, 1984; Higuchi, 1987; Guazzelli, 1993; Sanquetta et al., 1995; Teixeira et al., 2007; Souza \& Soares, 2013).

A técnica se baseia em probabilidades de ocorrência dos eventos que propiciam a dinâmica florestal, tais como: ingresso ou a regeneração natural, mortalidade ou a perturbação do dossel e o crescimento em classes de tamanho (Austregésilo et al., 2004; Teixeira et al., 2007). O Processo Markoviano é uma das técnicas mais conhecidas e empregadas no âmbito de processos estocásticos, sendo usada amplamente pela fácil aplicabilidade e menor quantidade de remedições necessárias para realizar a prognose (Vanclay, 1994; Nascimento et al., 2012).

$\mathrm{O}$ uso da técnica de modelagem matricial, bem como de técnicas de agrupamento ecológico de espécies, tem se mostrado proveitoso em diferentes trabalhos (Buogiorno \& Michie, 1980; Arce et al., 1997; Cunha et al., 2002; Canalez et al., 2006). Entretanto, a associação das duas técnicas de análise na dinâmica de grupos ecológicos em populações florestais é discutida por poucos trabalhos (Nappo et al., 2005), sendo a maioria dos estudos aplicados em florestas primárias e para todas ou uma espécie em particular (Enright \& Ogden, 1979; Sanquetta et al., 1995; Teixeira et al., 2007; Dalla Lana et al., 2015) 
O presente estudo tem como objetivo projetar a estrutura diamétrica de um fragmento de Floresta Ombrófila Mista, avaliando os aspectos sucessionais da dinâmica florestal pela prognose da distribuição diamétrica dos grupos ecológicos.

\section{MATERIAL E MÉTODOS}

\section{1. Área de estudo e coleta de dados}

O presente estudo foi realizado em um fragmento de Floresta Ombrófila Mista, localizado no Campus III da Universidade Federal do Paraná, em Curitiba, Paraná. O fragmento possui área total de 15,4 ha, situado entre as coordenadas geográficas $25^{\circ} 26^{\prime} 50^{\prime \prime}$ e $25^{\circ} 27^{\prime} 33^{\prime \prime}$ S e $49^{\circ} 14^{\prime} 16^{\prime \prime}$ e $49^{\circ} 14^{\prime} 33^{\prime \prime} \mathrm{W}$, com altitude variando de 890 a 915 m sobre o nível do mar (Nascimento et al., 2015). O clima da região, segundo a classificação de Köppen, é predominantemente Cfb, subtropical úmido mesotérmico, com temperaturas médias anuais em torno de $17^{\circ}$ e precipitação média anual chegando a $1500 \mathrm{~mm}$, bem distribuídos ao logo do ano (Machado et al., 2013). De acordo com Machado et al. (2013), o fragmento em questão encontra-se em estágio secundário de sucessão ecológica, devido a processos exploratório passado. Por se tratar de uma área urbana, a antropização é evidente, principalmente nas bordas do fragmento em contato com as vias públicas.

Os dados são provenientes de inventário florestal contínuo, configurado em censo florestal, cujas remedições são efetuadas sucessivamente a cada três anos, sempre no mês de julho, sendo: 20092012 e 2015. Para este estudo foram utilizadas as medições dos anos 2009 e 2012, sendo a de 2015 reservada para a validação das projeções. $\mathrm{O}$ intervalo de três anos é comumente empregado em estudos de dinâmica em florestas mistas secundárias (Stepka et al., 2010; Ebling et al., 2012), e devido à acuracidade das estimativas é adequado ao ritmo de crescimento dessas florestas. Toda a extensão do fragmento foi subdividida em blocos de $50 \times 50 \mathrm{~m}$, em seguida mensurados todos os indivíduos arbóreos que possuíam diâmetro a altura do peito (medido a $1,3 \mathrm{~m}$ do solo), superior ou igual a $10 \mathrm{~cm}$. Todos os indivíduos contemplados pelo critério de inclusão foram identificados em nível de espécie, georreferenciados e enumerados conforme sua disposição em cada bloco por meio de uma plaqueta metálica de numeração.

\subsection{Análise dos dados}

A classificação ecológica das espécies foi feita com base na proposta de Swaine \& Whitmore (1988), em que o agrupamento de características ecológicas similares é feito em apenas dois grupos: pioneiras - espécies dependentes de luz solar direta - e não pioneiras - composto por espécies tolerantes a sombra.

A construção da Matriz foi realizada por meio da quantificação dos processos de permanência da árvore na classe de diâmetro, avanço de uma ou mais classes, perda via mortalidade e ingresso via regeneração natural. A fórmula matricial e as expressões usadas para os cálculos da Matriz de Transição estão contidas em Stepka et al. (2010). Foram utilizados intervalos de 5 e $10 \mathrm{~cm}$ para agrupamento dos dados para a análise do comportamento preditivo das matrizes de transição

As probabilidades de ocorrência de cada processo foram calculadas por simples proporção, dividindo-se respectivamente o número de árvores que mudaram ou permaneceram na mesma classe, pelo número total de árvores na classe no início do período de crescimento (Austregésilo et al., 2004; Souza \& Soares, 2013). A mortalidade foi obtida pela razão entre o número de indivíduos mortos no período observado, pelo total de indivíduos na primeira ocasião (Equação 1). Já o ingresso foi calculado considerando todas as árvores ausentes na primeira ocasião (Equação 2). Foram adotadas ainda três possibilidades de ingresso, correspondendo respectivamente ao avanço para 1, 2 ou 3 classes sucessivas.

$T M=T M /(N T-T R)$

$T R=T R /(N T-T M)$

Em que $T M=$ taxa de mortalidade entre duas ocasiões; $T R=$ taxa de recrutamento entre duas ocasiões; $\mathrm{e}$ $N T=$ número total de indivíduos observados no período.

Foram calculados o tempo de meia vida $\left(\mathrm{t}_{1 / 2 ;}\right.$ Equação 3$)$ e o período de duplicação ( $\mathrm{t}_{2 ;}$ Equação 4), que correspondem, respectivamente, ao tempo em anos que a floresta ou um grupo de espécies leva para reduzir sua densidade pela metade e o tempo necessário para a duplicação da densidade (Korning \& Balslev, 1994; Ebling et al., 2013). A taxa anual de mortalidade foi calculada por meio da Equação 5. Esses períodos podem auxiliar a projeção da dinâmica da floresta, por meio da quantificação dos elementos mortalidade e ingresso durante duas ocasiões, evidenciando assim tendências futuras na sucessão florestal. 
$t \frac{1}{2}=\frac{\operatorname{Ln} 1 / 2}{\operatorname{Ln}(1-r)}$

$t_{2}=\frac{\operatorname{Ln} 2}{\operatorname{LN}(1+r)}$

$r=\frac{C 1}{C 0}^{\frac{1}{t}}-1$

Em que $L n=$ logaritmo de base neperiana; $r=$ taxa anual de mortalidade; $t=$ período de tempo analisado; $\mathrm{CO}=$ densidade na primeira ocasião, e; $C 1=$ densidade na segunda ocasião.

Inicialmente a Matriz de Transição foi construída por meio das medições realizadas nos anos de 2009 e 2012, sendo a estrutura diamétrica projetada para o ano de 2015. Com base na remedição feita em 2015, a precisão das projeções por grupo ecológico e para toda floresta foi avaliada pelo teste de aderência de Kolmogorov-Smirnov ao nível de significância de $1 \%$ e $5 \%$

Foi avaliado o efeito dos diferentes intervalos de classe diamétrica na aderência das projeções por meio do valor D de Kolmogorov-Smirnov e do erro-padrão da estimativa (Syx\%). De forma complementar, foi realizada a análise gráfica das distribuições estimadas pelas observadas, para a verificação da precisão das projeções. Por fim, foram feitas projeções sucessivas para os anos de 2018, 2021, 2024 e 2027, para avaliar as tendências de sucessão da floresta por meio do comportamento dos grupos ecológicos.

\section{RESULTADOS E DISCUSSÃO}

\subsection{Matriz de transição}

O número de indivíduos total e por grupo ecológico nos anos observados, o tempo de meia vida e duplicação, bem como as taxas de recrutamento e mortalidade, calculadas por grupo e para toda floresta e o teste de KS estão contidos na Tabela 1 . Observou-se nos períodos analisados uma tendência de redução da densidade total da floresta e dos grupos ecológicos. O grupo de espécies pioneiras apresentou dinâmica mais intensa, refletindo nas maiores taxas de recrutamento e mortalidade encontrada. Este fato pode ser explicado devido às espécies pioneiras apresentarem como características ecológicas o rápido crescimento e curto ciclo de vida (Swaine \& Whitmore, 1988; Piña-Rodrigues et al., 1990), conferindo intensa movimentação à dinâmica estrutural da floresta.

O teste de aderência de Kolmogorov-Smirnov (Tabela 1) evidenciou aderência entre os valores reais observados e as projeções em ambos os intervalos de classe avaliados, indicando a eficiência da Matriz de Transição para avaliação da sucessão florestal no fragmento de floresta ombrófila mista estudado. No entanto, os menores intervalos de classe forneceram as estimativas mais acuradas, sendo os mais adequados para a projeção.

Conforme constatado no presente estudo, Ebling et al. (2012), avaliando a acuracidade de métodos de projeção da distribuição diamétrica, mencionaram que os menores intervalos de classe também forneceram as estimativas mais precisas tanto no método da Matriz de Transição quanto no da razão de movimentação. Arce et al. (1997) citaram que a escolha inadequada das amplitudes de classe diamétrica pode conduzir a uma Matriz de Transição imprópria e de difícil interpretação. Avaliando o desempenho dessa técnica para simulações precoces do crescimento em Pinus taeda, esses autores recomendaram amplitudes compatíveis ao espaçamento, evitando assim interpretações equivocadas.

Comparando métodos de prognose da estrutura diamétrica, Austregésilo et al. (2004) constataram que,

Tabela 1. Frequência de indivíduos observados, dinâmica e projeção dos tempos de duplicação e meia vida por grupo ecológico nos anos de medição analisados.

Table 1. Frequency of observed individuals, dynamic and projection of doubling times and half-life by ecological group in the years of measurement analyzed.

\begin{tabular}{|c|c|c|c|c|c|c|c|c|c|}
\hline \multirow{2}{*}{$\begin{array}{c}\text { Grupo } \\
\text { Ecológico }\end{array}$} & \multicolumn{3}{|c|}{ Ind./ha. } & \multirow{2}{*}{$\begin{array}{l}\text { Tempo de } \\
\text { Meia Vida }\end{array}$} & \multirow{2}{*}{$\begin{array}{c}\text { Tempo de } \\
\text { Duplicação }\end{array}$} & \multirow{2}{*}{$\begin{array}{c}\text { Taxa de } \\
\text { Mortalidade }\end{array}$} & \multirow{2}{*}{$\begin{array}{c}\text { Taxa de } \\
\text { Recrutamento }\end{array}$} & \multicolumn{2}{|c|}{ Teste de K-S } \\
\hline & 2009 & 2012 & 2015 & & & & & $5 \mathrm{~cm}$ & $10 \mathrm{~cm}$ \\
\hline Pioneiras & 257 & 250 & 242 & 66 & - & 10,05 & 7,05 & ** & * \\
\hline $\begin{array}{l}\text { Não } \\
\text { pioneiras }\end{array}$ & 394 & 386 & 377 & - & 102 & 6,70 & 5,08 & ** & ** \\
\hline Floresta & 651 & 636 & 619 & 84 & - & 8,01 & 5,84 & $* *$ & $* *$ \\
\hline
\end{tabular}

* significativo ao nível de probabilidade de $99 \%$; ${ }^{* *}$ significativo ao nível de probabilidade de $95 \%$. 
para a Matriz de Transição, as projeções diferiram estatisticamente das estruturas reais. Assim, esses autores concluíram que a técnica foi eficiente apenas para projeção da densidade e não para a projeção da distribuição de diâmetros.

\subsection{Projeções sucessivas}

Após a validação da primeira projeção, foram realizadas projeções sucessivas para avaliar as tendências de sucessão da floresta, utilizando o intervalo de classes de $5 \mathrm{~cm}$, selecionado como o mais adequado para as estimativas. Por meio da evolução da distribuição diamétrica (Tabela 2) e da análise gráfica das projeções (Figura 1), alguns padrões foram evidenciados quanto ao comportamento dos grupos ecológicos e da floresta como um todo. Em todas as análises, a tendência de J-invertido foi mantida nas projeções, o que sugere uma boa regeneração natural em fluxo contínuo, caracterizando a perpetuidade das populações florestais conforme o avanço sucessional (Harper, 1990; Paula et al., 2004).

Outra tendência explicitada foi a redução da densidade conforme a sucessão no tempo. Essa tendência é conhecida na ciência florestal como Lei do Autodesbaste (Johnson et al., 2002). O autodesbaste é um processo natural caracterizado pela redução do número de árvores devido à mortalidade (Meyer, 2011) e intensificado pela competição por espaço e recursos. Segundo Ebling et al. (2013), essa redução, associada ao crescimento da população remanescente, indica um avanço sucessional. Nesse sentido, Gentry \& Terborgh (1990) afirmaram que o autodesbaste está relacionado às fases avançadas do ciclo silvogenético, indicando a maturidade da floresta. No entanto, essa tendência foi mais intensa nas primeiras classes e devido ao grande número de indivíduos presentes, contribuiu expressivamente para a redução da densidade total da floresta. A dinâmica mais intensa nas menores classes é reflexo da intensa movimentação causada pela mortalidade e ingresso (Stepka et al., 2010; Lana et al., 2015), sobretudo pela influência da regeneração natural contínua. Para a floresta e para o grupo de espécies não pioneiras, as classes superiores a $20 \mathrm{~cm}$ apresentaram um aumento na frequência, em razão do avanço de indivíduos das primeiras classes e do movimento entre classes, caracterizando o crescimento dos componentes remanescentes. Quanto às pioneiras, esse aumento foi mais evidente nas classes superiores a $30 \mathrm{~cm}$.

A mesma tendência de redução mais acentuada nas primeiras classes, com leve aumento nas demais classes com o passar dos anos, foi observada por Stepka et al. (2010). Já Lana et al. (2015) encontraram tendências opostas para um trecho de floresta ombrófila mista no Paraná. Esses autores observaram pequenos aumentos nas primeiras classes e redução nas maiores. Essas diferenças entre a dinâmica das florestas, possivelmente ocorre por causa do balanço entre as taxas de mortalidade e recrutamento. Stepka et al. (2010), conforme o presente estudo, encontraram taxas de mortalidade superiores às de ingresso, ao contrário de Lana et al. (2015), que citam as taxas de recrutamento sempre superiores às de mortalidade. A dominância da mortalidade em relação ao ingresso pode ser reflexo da maior competição das árvores,

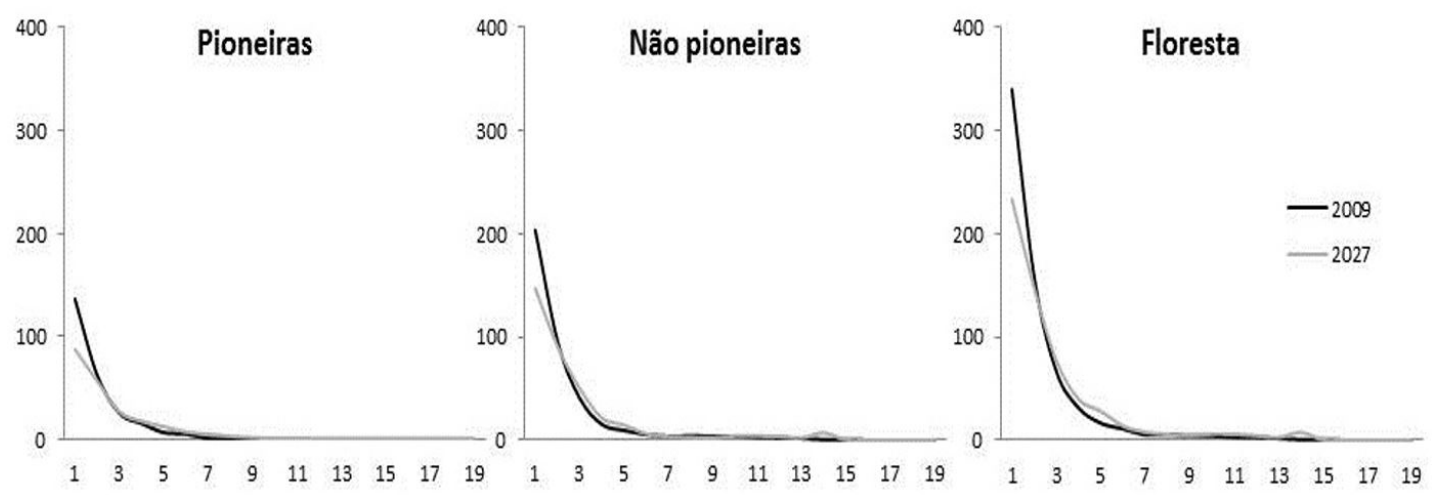

Figura 1. Tendência da distribuição diamétrica entre os anos de 2009 e 2027, adotando o intervalo mais adequado para a projeção (Classe de $5 \mathrm{~cm}$ ).

Figure 1. Tendency in diameter distribution between the years 2009 and 2027, adopting the most appropriate classe interval for the projection $(5 \mathrm{~cm})$. 
$\circ$

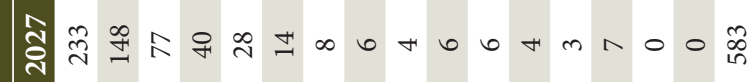

ปี่

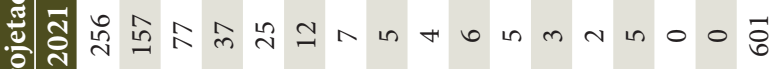
I

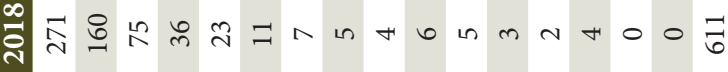

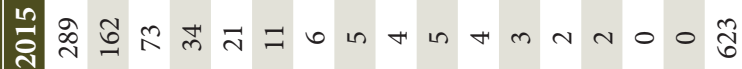

产

हี่

도

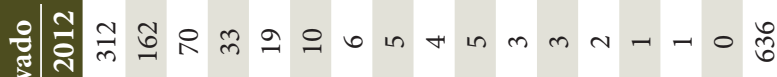

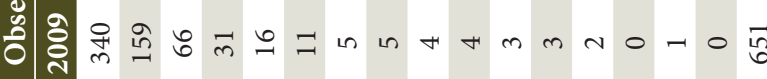

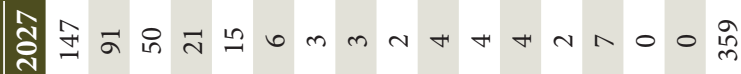

预约

ปี่

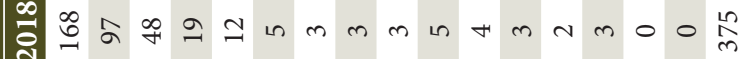

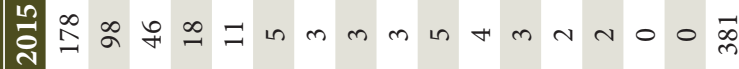

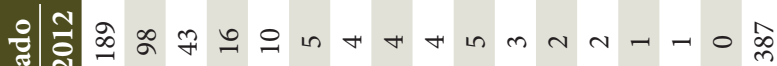

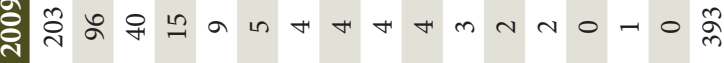

ปิ

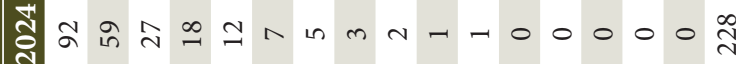


principalmente nas menores classes (Stepka et al., 2010), onde os indivíduos juvenis podem estar ainda em fase de adaptação. Para Nappo et al. (2005), o autodesbaste na primeira classe é uma consequência da estruturação do povoamento, que afeta a disponibilidade de recursos conforme a sucessão.

Considerando o comportamento dos grupos ecológicos em relação à floresta, a redução na densidade foi mais intensa no grupo das pioneiras. Já as não pioneiras, mesmo apresentando redução na densidade, revelam um leve aumento na frequência relativa, corroborando o encontrado no cálculo dos tempos de meia vida e tempo de duplicação (Tabela 1). Esta constatação sugere uma tendência de substituição gradual das espécies pioneiras restritas às clareiras e bordas por não pioneiras, indicando um avanço sucessional da floresta. Segundo Paula et al. (2004), a menor representatividade florística das pioneiras expressa um estádio intermediário de desenvolvimento sucessional da floresta. Com a ausência de impactos antrópicos como o corte de madeiras, ocorreu menor oportunidade para o surgimento e desenvolvimento de pioneiras, exceto nas clareiras naturais e bordas, favorecendo o estabelecimento de não pioneiras, conforme o constatado por Pagano et al. (1995).

A tendência de substituição deve-se às pioneiras crescerem mais rápido e entrarem em competição mais cedo em relação às não pioneiras, que são progressivamente substituídas pelas clímax, que cresçem lentamente (Whitmore, 1989). Essa tendência também foi demonstrada por Nascimento (2012), que provou a substituição contínua e gradual na dominância de espécies pioneiras por não pioneiras em uma floresta ombrófila de terra firme na Amazônia Central. Para a floresta ombrófila mista, esse comportamento de substituição também é citado. Silva (2013), estudando os aspectos sucessionais de bracatingais em florestas mistas, citou que a espécie pioneira Mimosa scabrela Benth. tem importância estrutural gradualmente substituída ao longo do processo de sucessão, caracterizado pelo aumento na diversidade e riqueza de espécies. Bormann \& Likens (1979) apud Whitmore (1989), afirmou que em geral a composição florística da paisagem tende a permanecer estável, com ocorrência de alterações na configuração e composição das manchas ao longo do tempo.
Whitmore (1989) mencionou que os processos de sucessão em florestas naturais variam de acordo com a condição à qual estão propensos, determinando o predomínio de espécies pioneiras ou não pioneiras no mosaico estrutural da floresta. De acordo com esse autor, o predomínio de um determinado grupo ecológico depende do tamanho das clareiras típicas da dinâmica da floresta, que configura o mosaico sucessional local. Outro fator relevante: as espécies pioneiras e não pioneiras podem ocupar o mesmo ambiente na floresta, e essa colonização simultânea produz mudanças sutis na composição (Brokaw \& Schemer, 1989 apud Whitmore, 1989; Egler, 1954 apud Whitmore, 1989). Essa leve diferença na composição foi observada no presente estudo, apresentando uma dinâmica que corrobora com a teoria ecológica.

Diversos fatores bióticos e abióticos determinam uma composição florística específica para um certo estágio e idade de desenvolvimento, influenciando a recuperação estrutural e funcional da vegetação (Guariguata \& Ostertag, 2001). Esses autores acrescentaram que, embora a compreensão dos processos de sucessão em florestas seja possível, é muito dificil prever os padrões de substituição de espécies, devido à interação de diversos fatores.

As florestas tropicais naturais se autorregulam de acordo com os processos locais de mortalidade, crescimento e regeneração, em que as árvores perdidas são continuamente substituídas, até atingir um equilíbrio ou estado dinâmico estacionário (Swaine et al., 1987). O conhecimento dessas tendências de evolução e sucessão florestal são imprescindíveis para apoiar a tomada de decisão quanto à conservação e manejo dos recursos florestais. Compreender os mecanismos de sucessão torna-se fundamental para o desenvolvimento de estratégias de conservação da floresta tropical, visto que são consideradas as florestas do futuro (Wright, 2005; Quesada et al., 2009).

Os estudos desenvolvidos no Brasil com a técnica Matriz de Transição evidenciaram prós e contras advindos de seu emprego, por exemplo: os períodos de projeção aceitam apenas valores múltiplos dos períodos de remedição (Ebling et al., 2012); as probabilidades de movimentação são fixas (Schneider \& Finger, 2000; Lana et al., 2015); fácil aplicação, pois não envolve variáveis de difícil acesso como idade e índice de sítio (Higuchi, 1987; Sanquetta et al., 1996); possibilidade de 
realizar projeções com apenas duas medições sucessivas (Teresczcuch et al., 2007).

Na Matriz de Transição, as projeções são dependentes da situação em que a floresta se encontrava por ocasião da coleta de dados, definido como propriedades Markovianas (Schneider \& Finger, 2000). Neste estudo, foram observados estados absorventes, definidos pela probabilidade de transição entre uma classe e a classe subsequente ser igual a zero. Os estados absorventes são uma desvantagem apresentada por esse método de projeção, observado por outros pesquisadores que o utilizaram, como: Austregésilo et al. (2004), Stepka et al. (2010) e Lana et al. (2015). Para a floresta e para as espécies não pioneiras, esse estado ocorreu na classe 85, e para as pioneiras, na classe 80 . Esses estados podem interferir nas projeções, logo, as análises para longo prazo devem ser realizadas com cautela. $\mathrm{O}$ efeito desses pode ser menor considerando as espécies pioneiras, as quais raramente apresentam diâmetros superiores a $80 \mathrm{~cm}$.

Bons resultados para essa técnica foram obtidos nos estudos realizados por Lana et al. (2015), Stepka et al. (2010), Teixeira et al. (2007), Cunha et al. (2002), Sanquetta et al. (1996), Higuchi (1987). No entanto, quando comparada a outras técnicas, a Matriz de Transição pode apresentar resultados inferiores, como constatado por Ebling et al. (2012), utilizando também a Razão de Movimentos.

Para estudos ecológicos com enfoque sucessional, a aplicação de técnicas de prognose ainda é pouco explorada, sendo escassos os estudos para as florestas naturais brasileiras. Orellana (2014), avaliando a sucessão florestal de grupos ecológicos por meio de um modelo de árvore individual, evidenciou características do processo de sucessão, representada por flutuações na densidade e área basal dos grupos de espécies. Com o objetivo de caracterizar melhor o processo de sucessão da regeneração natural, Nappo et al. (2005) aplicaram a Matriz de Transição, realizando a prognose para toda regeneração e por grupo ecológico. Esses autores encontraram resultados satisfatórios com a técnica, enfatizando sua utilidade e praticidade para avaliação do processo de sucessão. Esses estudos demonstraram-se eficazes para avaliar a dinâmica da floresta, principalmente devido à análise dos grupos ecológicos, fornecendo importantes informações sobre a sucessão das florestas naturais, norteando, assim, ações de manejo e conservação de seus recursos.

\section{CONCLUSÃO}

A aderência ao conjunto de dados e os valores satisfatórios das estatísticas de precisão evidenciaram a eficiência da Matriz de Transição para a prognose da estrutura diamétrica e da densidade total. A técnica propiciou resultados satisfatórios para a projeção da estrutura diamétrica dos grupos ecológicos, servindo como ferramenta útil para avaliação da sucessão florestal em fragmentos de floresta ombrófila mista.

A leve redução da frequência relativa das espécies pioneiras, seguida do aumento gradual da frequência das não pioneiras, sugere um avanço sucessional do fragmento em questão, partindo do predomínio de estágios intermediários no mosaico sucessional, para estágios avançados. A tendência de autodesbaste observada ratifica o avanço sucessional, pois é característica da dinâmica estrutural de florestas em fases avançadas do ciclo de sucessão.

\section{STATUS DA SUBMISSÃO}

Recebido: 5 maio, 2016

Aceito: 1 out., 2016

\section{AUTOR(ES) PARA CORRESPONDÊNCIA}

\section{Vinicius Cysneiros}

Departamento de Ciências Florestais, Universidade Federal do Paraná - UFPR, Rua Lothário Meisser, 632, CEP 80060-000, Curitiba, PR, Brazil

e-mail: vccysneiros.florestal@gmail.com

\section{REFERENNCIAS}

Alder D. Forest volume estimation and yield prediction. Roma: FAO Forestry Paper; 1980. vol. 2.

Arce JE, Pizatto W, Sanquetta CR, Wendling JLG, Maestri R. Utilização das matrizes de transição na avaliação e simulação precoces do crescimento de povoamentos de Pinus taeda L. Floresta 1997; 27(1): 83-98.

Austregésilo SL, Ferreira RLC, Silva JÁ, Souza AL, Meunier IMJ, Santos ES. Comparação de métodos de prognose da estrutura diamétrica de uma Floresta Estacional Semidecidual 
secundária. Revista Árvore 2004; 28(2): 227-232. http:// dx.doi.org/10.1590/S0100-67622004000200009.

Buogiorno J, Michie BC. A matrix model of unever-aged forest management. Forest Science 1980; 26: 609-625.

Canalez GG, Corte APD, Sanquetta CR. Dinâmica da estrutura da comunidade de Lauráceas no período 1995 2004 em uma floresta de araucária no sul do estado do Paraná, Brasil. Ciência Florestal 2006; 16(4): 357-367. http://dx.doi.org/10.5902/198050981917.

Cunha US, Machado AS, Figueiredo A Fo, Sanquetta CR. Predição da estrutura diamétrica de espécies comerciais de terra firme da Amazônia por meio de Matriz de Transição. Ciência Florestal 2002; 12(1): 109-122. http:// dx.doi.org/10.5902/198050981705.

Ebling AA, Péllico S No, Watzlawick LF, Miranda ROV, Abrão SF. Ecologia e projeção diamétrica de três grupos arbóreos em remanescente de floresta ombrófila mista em São Francisco de Paula, RS. Floresta 2013; 43(2): 261-270. http://dx.doi.org/10.5380/rf.v43i2.28604.

Ebling AA, Watzlawick LF, Rodrigues AL, Longhi SJ, Longhi RV, Abrão SF. Acuracidade da distribuição diamétrica entre métodos de projeção diamétrica em Floresta Ombrófila Mista. Ciência Rural 2012; 42(6): 1020-1026. http://dx.doi. org/10.1590/S0103-84782012000600011.

Enright N, Ogden J. Applications of transition matrix models in forest dynamics: Araucaria in Papua, New Guinea e Nothofagus in New Zealand. Australian Journal of Ecology 1979; 4(4): 3-23. http://dx.doi.org/10.1111/j.1442-9993.1979. tb01195.x.

Gandolfi S, Leitão HF Fo, Bezerra CLE. Levantamento florístico e caráter sucessional das espécies arbustivo arbóreas de uma floresta mesófila semidecídua no município de Guarulhos, SP. Revista Brasileira de Biologia 1995; 55(4): 753-767.

Gentry AH, Terborgh J. Composition and dynamics of the Cosha Cashu mature: floodplain forest. In: Gentry AH. Four neotropical rainforests. New Haven: Yale University Press; 1990.

Guariguata MR, Ostertag R. Neotropical secondary forest succession: changes in structural and functional characteristics. Forest Ecology and Management 2001; 148(1-3): 185-206. http://dx.doi.org/10.1016/S03781127(00)00535-1.

Guazzelli MR. Teoria e prática sobre as cadeias de Markov. Ambiente 1993; 7(1): 45-51.

Harper JL. Population biology plants. London: Academic; 1990.

Higuchi N. Short-term growth of a nun disturbed tropical moist forest in the Brazilian Amazon [tese]. Michigan: Michigan State University; 1987.

Johnson PS, Shifley SR, Rogers R. The ecology and silviculture of oaks. New York: CABI Publishing; 2002.
Korning J, Balslev H. Growth and mortality of trees in Amazonian tropical rain forest in Ecuador. Journal of Vegetation Science 1994; 4(1): 77-86. http://dx.doi. org/10.2307/3235641.

Lana MD, Péllico S No, Corte APD, Sanquetta CR, Ebling AA. Prognose da estrutura diamétrica em Floresta Ombrófila Mista. Floresta e Ambiente 2015; 22(1): 71-78. http://dx.doi.org/10.1590/2179-8087.072914.

Machado AS, Zamin NT, Nascimento RGM, Augustynczyk ALD, Menegazzo CS. Comparação dos parâmetros fitossociológicos entre três estratos de um fragmento de floresta ombrófila mista. Cerne 2013; 19(3): 365-372. http://dx.doi.org/10.1590/S0104-77602013000300002.

Maciel MNM, Watzlawick LF, Schoeniger ER, Yamaji FM. Classificação ecológica de espécies arbóreas. Acadêmica 2003; 1(2): 69-78.

Meyer EA. Densidade de árvore por diâmetro na Floresta Estacional Decidual no Rio Grande do Sul [dissertação]. Santa Maria: Universidade Federal de Santa Maria; 2011.

Nappo ME, Griffith JJ, Martins SV, Marco P Jr, Souza AL, Oliveira AT Fo. Dinâmica da estrutura diamétrica da regeneração natural de espécies arbóreas e arbustivas no sub-bosque de povoamento puro de Mimosa scabrella Bentham, em área minerada, em Poços de Caldas, MG. Revista Árvore 2005; 29(1): 35-46. http://dx.doi.org/10.1590/ S0100-67622005000100005.

Nascimento RG. Modelagem e prognose da produção de uma floresta tropical úmida de terra firme na Amazônia Central [dissertação]. Curitiba: Universidade Federal do Paraná; 2012.

Nascimento RGM, Machado AS, Figueiredo A Fo, Higuchi N. Modelo de projeção por classe diamétrica para florestas nativas: enfoque na função probabilística de Weibull. Pesquisa Florestal Brasileira 2012; 32(70): 209-219.

Nascimento RGM, Silva LCR, Barbeiro LSS, Wojciechowski JC, Péllico S No, Machado SA. Efeito da árvore marginal nos estimadores populacionais obtidos por métodos de amostragem de área variável. Cerne 2015; 21(1): 125-131. http://dx.doi.org/10.1590/01047760201521011266.

Odum EP. The strategy of ecosystem development. Science 1969; 164(3877): 262-270. PMid:5776636. http://dx.doi. org/10.1126/science.164.3877.262.

Orellana E. Sucessão florestal, regimes de manejo e competição avaliados com modelos de árvore individual dependente e independente da distância em um fragmento de Floresta de Araucária no Sul do Brasil [tese]. Curitiba: Universidade Federal do Paraná; 2014.

Pagano SN, Leitão HP Fo, Cavassan O. Variação temporal da composição florística e estrutura fitossociológica de uma floresta mesófila semidecídua - Rio Claro - Estado de São Paulo. Revista Brasileira de Biologia 1995; 2: 241-258.

Paula A, Silva AF, Marco P Jr, Santos FAM, Souza AL. Sucessão ecológica da vegetação arbórea em uma Floresta 
Estacional Semidecidual, Viçosa, MG, Brasil. Acta Botanica Brasílica 2004; 18(3): 407-423. http://dx.doi.org/10.1590/ S0102-33062004000300002.

Pillar VD. Dinâmica temporal da vegetação [online] Porto Alegre: UFRGS; 1994 [citado em 2016/02/14]. Disponível em http://ecoqua.ecologia.ufrgs.br/ arquivos/Reprints\&Manuscripts/Manuscripts\&Misc/8_ Sucessao_94Nov21.pdf.

Piña-Rodrigues FCM, Costa LGS, Reis A. Estratégias de estabelecimento de espécies arbóreas e o manejo de florestas tropicais. In: Anais do $6^{\circ}$ Congresso Florestal Brasileiro; 1990; Campos do Jordão. São Paulo: Sociedade Brasileira de Silvicultura; 1990. p. 676- 684.

Quesada M, Sanchez-Azofeife GA, Alvarez-Añorve M, Stoner KE, Avila-Cabadilla L, Calvo-Alvarado J et al. Succession and management of tropical dry forests in the Americas: review and new perspectives. Forest Ecology and Management 2009; 258(6): 1014-1024. http://dx.doi. org/10.1016/j.foreco.2009.06.023.

Richards PW. The tropical rain forest: an ecological study. Cambridge: Cambridge University Press; 1957.

Sanquetta CR, Angelo H, Brena DA, Mendes JB. Predição da distribuição diamétrica, mortalidade e recrutamento de floresta natural com matriz Markoviana de potência. Floresta 1995; 24(1/2): 23-26.

Sanquetta CR, Brena DR, Angelo H, Mendes JB. Matriz de transição para simulação da dinâmica de florestas naturais sob diferentes intensidades de corte. Ciência Florestal 1996; 6(1): 65-78.

Schneider PR, Finger CAG. Manejo sustentado de florestas inequiâneas heterogêneas. Santa Maria: UFSM, 2000.

Shuggart HH. A theory of forest dynamics: the ecological for succession model. New York: Springer-Verlag, 1984.

Silva LCR. Estrutura e florística de bracatingais da região metropolitana de Curitiba, Paraná: um enfoque sucessional. [dissertação]. Curitiba: Universidade Federal do Paraná; 2013.

Souza AL, Soares CPB. Florestas nativas: estrutura, dinâmica e manejo. Viçosa: Editora UFV; 2013.
Stepka TF, Dias AN, Figueiredo Filho A, Machado SA, Sawczuk RA. Prognose da estrutura diamétrica de uma Floresta Ombrofila Mista com os métodos razão de movimentos e matriz de transição. Pesquisa Florestal Brasileira 2010; 30(64): 327-335. http://dx.doi. org/10.4336/2010.pfb.30.64.327.

Swaine MD, Lieberman D, Pultz FE. The dynamics of tree populations in tropical forest: a review. Journal of Tropical Ecology 1987; 3(4): 359-366. http://dx.doi.org/10.1017/ S0266467400002339.

Swaine MD, Whitmore TC. On the definition of ecological species groups in tropical rain forests. Vegetation 1988; 75(1/2): 81-86. http://dx.doi.org/10.1007/BF00044629.

Teixeira LM, Chambers JQ, Silva AR, Lima AJN, Carneiro VMC, Santos J et al. Projeção da dinâmica da floresta natural de Terra-firme, região de Manaus - AM, com uso da cadeia de transição probabilistic de Markov. Acta Amazonica 2007; 37(3): 377-384. http://dx.doi.org/10.1590/ S0044-59672007000300009.

Tereschcuch SM, MacDonagh PM, Oliveira AJ, Rivero LE, Bulfe NML. Prediccíon de la estructura diamétrica de especies comerciales de um bosque subtropical por medio de matrices de transicíon. Floresta 2007, 37(1): 71-80.

Vanclay JK. Modelling Forest growth and yield: applications to mixed tropical forests. Wallingford: CAB International, 1994.

Whitmore TC. Canopy gaps and two major groups of forest trees. Ecology 1989; 70(3): 536-538. http://dx.doi. org/10.2307/1940195.

Whitmore TC. Gaps in the forest canopy. In: Tomlinson Z. Tropical trees as living systems. London: Cambridge University Press; 1978.

Whitmore TC. Tropical Rain Forest dynamics and its implications for management. In: Gomes-Pompa A, Whitmore TC, Hadley M. Rain forest regeneration and management. Paris: UNESCO; 1990.

Wright SJ. Tropical forests in a changing environment. Trends in Ecology \& Evolution 2005; 20(10): 553-560. PMid:16701434. http://dx.doi.org/10.1016/j.tree.2005.07.009. 